\title{
DECOMPOSING THE BROWNIAN PATH
}

BY DAVID WILLIAMS

Communicated by H. P. McKean, February 20, 1970

1. Starred references are to Itô and McKean [3], the terminology of which is used here.

Because of the method of time substitution (Chapter $5^{*}$ ), results on the structure of the Brownian path generalise easily to provide analogous results for arbitrary diffusions. The generalisations of the results described here take simple forms in terms of scale function, speed measure and generator. These generalisations and their proofs will appear elsewhere.

Notation. $\mathrm{A} \mathrm{BM}^{0}$ is a (one-dimensional) Brownian motion starting at 0 . By a $\mathrm{BES}_{\mathbf{k}}^{0}$ we mean a ' $k$-dimensional' Bessel process starting at 0 , i.e., a continuous random function identical in law to the radial part of a $k$-dimensional Brownian motion which starts at 0. See $\$ 2.7 *$. Introduce the $k$-dimensional Bessel bridge $\mathrm{BES}(k) \mathrm{BR}$ as a random function on the parameter set $[0,1]$ identical in law to

$$
\operatorname{tr}_{k}[(1-t) / t]
$$

where $r_{k}$ is a $\mathrm{BES}_{k}^{0}$. Compare the 'Brownian bridge' in Billingsley [1].

2. Now let $w=\{w(t): t \geqq 0\}$ be a $\mathrm{BM}^{0}$ and put

$$
\tau=\inf \{s: w(s)=1\} .
$$

THEOREM 1. If $r_{3}$ is a $\mathrm{BES}_{3}^{0}$, then the random functions

$$
\{1-w(\tau-t): 0 \leqq t<\tau\} \quad \text { and } \quad\left\{r_{3}(t): 0 \leqq t<\sup \left[s: r_{3}(s)=1\right]\right\}
$$

are identical in law.

Next, put

$$
\begin{aligned}
& \sigma=\sup \{s: s<\tau ; w(s)=0\}, \\
& \alpha=\sup \{w(s): s<\sigma\} .
\end{aligned}
$$

Lemma 1. With probability one, there is precisely one value $\rho$ in $(0, \sigma)$ such that $w(\rho)=\alpha$.

Theorem 2 provides a type of decomposition of the Brownian path which proves useful.

AMS 1968 subject classifications. Primary 6060, 6062.

Key words and phrases. Brownian motion, Bessel process, local time. 
THEOREM 2. On a suitable probability triple, set up four independent random elements: a random variable $\alpha^{*}$ uniformly distributed on $(0,1)$, $a \mathrm{BM}^{0} w^{*}, a \mathrm{BES}_{3}^{0} r_{3}^{*}$ and another $\mathrm{BES}_{3}^{0} r_{3}^{* *}$. Define

$$
\begin{aligned}
& \rho^{*}=\inf \left\{t: w^{*}(t)=\alpha^{*}\right\}, \\
& \sigma^{*}=\rho^{*}+\sup \left\{t: r_{3}^{*}(t)=\alpha^{*}\right\}, \\
& \tau^{*}=\sigma^{*}+\inf \left\{t: r_{3}^{* *}(t)=1\right\} .
\end{aligned}
$$

Put

$$
\begin{array}{llrl}
\tilde{w}(t) & =w^{*}(t), & & t<\rho^{*} ; \\
\tilde{w}(t) & =\alpha^{*}-r_{3}^{*}\left(t-\rho^{*}\right), & & \rho^{*} \leqq t<\sigma^{*} ; \\
\tilde{w}(t) & =r_{3}^{* *}\left(t-\sigma^{*}\right), & & \sigma^{*} \leqq t<\tau^{*} .
\end{array}
$$

Then $\left\{\tilde{w}(t): t<\tau^{*}\right\}$ and $\{w(t): t<\tau\}$ are identical in law.

3. Introduce $L(t, x)$ as twice the standard Brownian local time at $x$ before $t$ for $w$ so that (see $\$ 2.8^{*}$ ) with probability one, $L(t, x)$ is jointly continuous in $t$ and $x$ and

$$
\text { measure }\{s: s \leqq t ; w(s) \in A\}=\int_{A} L(t, x) d x
$$

simultaneously for every $t \geqq 0$ and every Borel set $A$.

Theorem 3 ( $\operatorname{RAy}[4])$. The equation

$$
r_{2}(x)^{2}=L(\tau, 1-x)
$$

defines a $\mathrm{BES}_{2}^{0} r_{2}$ on $\{x: 0 \leqq x \leqq 1\}$.

Theorems 1, 2 and 3 lead to the following result.

THEOREM 4. Set up four independent random elements: a random variable $\beta$ uniformly distributed on $(0,1), a \operatorname{BES}(2) \mathrm{BR} u_{2}$, and two $\mathrm{BES}_{2}^{0}$ random functions $r_{2}^{*}$ and $r_{2}^{* *}$. Then the family of random elements

$$
\{L(\rho, x), L(\sigma, x)-L(\rho, x), L(\tau, x)-L(\sigma, x): 0 \leqq x \leqq 1 ; \alpha\}
$$

is identical in law to the family

$$
\left\{r_{2}^{*}\left([\beta-x]^{+}\right)^{2}, r_{2}^{* *}\left([\beta-x]^{+}\right)^{2}, u_{2}(x)^{2}: 0 \leqq x \leqq 1 ; \beta\right\}
$$

where $s^{+}$denotes $\max (s, 0)$.

CoROllary. Let $r_{4}$ be a $\mathrm{BES}_{4}^{0}$. Then the random functions

$$
\{L(\sigma, x): 0 \leqq x \leqq 1\} \quad \text { and } \quad\left\{r_{4}\left([\beta-x]^{+}\right)^{2}: 0 \leqq x \leqq 1\right\}
$$

are identical in law. 
A direct probabilistic proof of Theorem 3 was given in Williams [5]. It is hoped that the methods outlined there together with the theorems of this note help towards an intuitive understanding of Ray's theory of local time and, in particular explain the appearance of the squared $\mathrm{BES}_{4}^{0}$ random function in equation (11c) of $\$ 2.8^{*}$. That equation should read

$$
\mathrm{t}\left(\mathfrak{m}_{1}, a\right)=(1-a)^{2} r_{4}\left[\frac{1}{1-a}-\frac{1}{1-b}\right]^{2} / 2
$$

$(b \leqq a \leqq 0)$, a result equivalent to the corollary to Theorem 4 .

Notes. 1. The unsigned scaled Brownian excursion $\left(\$ 2.9^{*}\right)$ is a $\mathrm{BES}(3) \mathrm{BR}$.

2. Theorem 1 explains the case ' $k=1$ ' of the Ciesielski-Taylor theorem [2]: the time spent by $\operatorname{BES}_{k+2}^{0}$ in $(0,1)$ is identical in law to the hitting time of 1 by $\mathrm{BES}_{\mathbf{k}}^{0}$.

3. The well-known Cameron-Martin formula:

$$
E \exp \left[-\theta \int_{0}^{1} w(t)^{2} d t\right]=(\operatorname{sech} \gamma)^{1 / 2} \quad\left(0<2 \theta=\gamma^{2}\right)
$$

follows immediately from Theorem 3 .

\section{REFERENCES}

1. P. Billingsley, Convergence of probability measures, Wiley, New York, 1968. MR 38 \#1718.

2. Z. Ciesielski and S. J. Taylor, First passage times and sojourn times for Brownian motion in space and the exact Hausdorff measure of the sample path, Trans. Amer. Math. Soc. 103 (1962), 434-450. MR 26 \#816.

3. K. Itô and H. P. McKean, Jr., Diffusion processes and their sample paths, Die Grundlehren der math. Wissenschaften, Band 125, Academic Press, New York and Springer-Verlag, Berlin, 1965. MR 33 \#8031.

4. D. Ray, Sojourn times of diffusion process, Illinois J. Math. 7 (1963), 615-630. MR 27 \#6306.

5. D. Williams, Markov properties of Brownian local time, Bull. Amer. Math. Soc. 75 (1969), 1035-1036.

University College, Swansea, Great Britain 